Published in final edited form as:

J Exp Soc Psychol. 2012 January ; 48(1): 399-402. doi:10.1016/j.jesp.2011.07.015.

\title{
Strangers in sync: Achieving embodied rapport through shared movements
}

\author{
Tanya Vacharkulksemsuk, M.A. ${ }^{a,}$ and Barbara L. Fredrickson, Ph.D. ${ }^{a}$ \\ Tanya Vacharkulksemsuk: tanyav@email.unc.edu; Barbara L. Fredrickson: blf@unc.edu \\ aUniversity of North Carolina, Chapel Hill, Department of Psychology, Davie Hall CB \#3270, \\ Chapel Hill, NC 27599
}

\section{Abstract}

This paper examines the emergence of behavioral synchrony among strangers in the context of self-disclosure, and their path in predicting interaction quality. Specifically, we hypothesize that behavioral synchrony mediates the direct effect of self-disclosure on the development of embodied rapport. Same-sex stranger pairs $(n=94)$ were randomly assigned to a videorecorded selfdisclosure or control condition, and afterward each member rated their social interaction. Following the procedure used by Bernieri, Reznick, \& Rosenthal (1988), two trained judges independently watched each video record and rated each pair interaction on behavioral synchrony. Bootstrapping analyses provide support for the hypothesized mediating effect of behavioral synchrony, which emerged as independent of the effects of self-other overlap and positive affect. The authors discuss implications of behavioral synchrony for relationship formation processes and the inevitable entwinement of behavior and judgments in light of embodied cognition.

\section{Keywords}

Synchrony; Self-disclosure; Rapport; Embodiment; Relationship formation

Knowing that quality social relationships are critical for personal health (e.g., Snyder \& Lopez, 2002), it is important to understand the interpersonal micro-moments-the "building blocks" - of successful relationship formation. Acts of self-disclosure with strangers, be it informally on a morning subway commute or formally at a structured event like speeddating, are a common step toward building social relationships. Turns out, a mechanism by which self-disclosure is effective involves the unspoken, shared physical movements of the interactants.

The present study employs behavioral coding of same-sex stranger dyads during instances of self-disclosure, and results point to the spontaneous act of people moving together in time and space as a key element that promotes quality interaction-namely, embodied rapport. Whereas rapport is traditionally defined by interactants' perceptions of positivity and mutuality (Tickle-Degnen \& Rosenthal, 1990), recent findings on embodied cognition

(C) 2011 Elsevier Inc. All rights reserved.

*Correspondence concerning this article should be addressed to Tanya Vacharkulksemsuk, UNCCH Department of Psychology, CB \#3270, Chapel Hill, NC 27599, tanyav@email.unc.edu.

Publisher's Disclaimer: This is a PDF file of an unedited manuscript that has been accepted for publication. As a service to our customers we are providing this early version of the manuscript. The manuscript will undergo copyediting, typesetting, and review of the resulting proof before it is published in its final citable form. Please note that during the production process errors may be discovered which could affect the content, and all legal disclaimers that apply to the journal pertain. 
suggest that the bodily aspects of rapport have thus far gone under-appreciated. Consistent with embodied cognition's thesis that people's perceptions and judgments reflect their own motor and bodily experiences (see Niedenthal, 2007), we propose that rapport reflects interactants' physically shared motions, emotions, and vitality. In particular, beyond perceptions of positivity and mutuality, we propose that shared feelings of vitality and aliveness - bodily sensations theorized to stem from shared movements (McNeill, 1995)_ are also keys element of what we here term embodied rapport. ${ }^{1}$

\section{Behavioral Synchrony: Moving Together toward Embodied Rapport}

General cultural observations suggest that behavioral synchrony fosters a sense of "oneness" that brings people together: religious activities, military drilling, and rituals among sports teams involve rich amounts of common rhythms to which people can move in synchrony (McNeill, 1995). Infant-caretaker dyads also illustrate how synchronous behaviors guide relational and social development (Feldman, 2007). Beginning around three-months of age, for example, when infants begin to recognize and respond to others' facial expressions, gaze, vocal, and touch patterns, they notably achieve synchrony with their caretakers: a dyadic and consequential dance between caretaker and infant in recognizing and reciprocating affective states emerges (Weinberg \& Tronick, 1996).

Behavioral synchrony is the coordination of movement that occurs between individuals during a social interaction, featuring similarity of (1) form, the manner and style of movements, and (2) time, the temporal rhythm of movements (Kimura \& Daibo, 2006). Early studies of behavioral synchrony utilized a coding procedure developed by Bernieri, Reznick, and Rosenthal (1988) based on the assumption that Gestalt qualities of behavioral synchrony are apparent and perceivable by human observers. As judged by observers, successful language acquisition results from behavioral synchrony between newborn infants' movements and adult speech patterns (Condon \& Sander, 1974), and increased rapport within teacher-student dyads stems from behavioral synchrony (Bernieri, 1988). More recently, experimental manipulations of synchrony show that it breeds compassion (Valdesolo \& DeSteno, 2011), cooperation (Wiltermuth \& Heath, 2009), affiliation (Hove \& Risen, 2009), emotional support satisfaction (Jones \& Wirtz, 2007), and even elevated pain thresholds (Cohen, Ejsmond-Frey, Knight, \& Dunbar, 2010).

We see instances of self-disclosure as a sensible starting point for exploring our questions about behavioral synchrony and embodied rapport among strangers, particularly given its well-known ability to generate social closeness.

\section{Self-disclosure: Opening Doors for Embodied Rapport}

Self-disclosure involves revealing and sharing personal information about oneself, including facts, anecdotes, opinions, and emotions, to another person (Jourard, 1959), and evidence suggests that self-disclosure promotes relationships through mechanisms of liking and positive affect (Collins \& Miller, 1994; Strong \& Aron, 2006). One way self-disclosure is experimentally induced is through Aron's closeness paradigm (Aron, Melinat, Aron, Vallone, \& Bator, 1997), wherein participants within dyads alternate asking and answering questions that progressively reveal more information-in other words, progressively selfdisclose. This paradigm has been successfully adapted by several researchers (e.g., Kashdan \& Roberts, 2004), and has been shown to produce neuroendocrine changes even when used in abbreviated form (Brown, et al, 2009). Consistent with evidence of self-disclosure's

\footnotetext{
${ }^{1}$ Dutton and Heaphy (2003), working within organizational studies, identify the construct we here term embodied rapport as highquality connections.
} 
benefits for relationships, we seek to demonstrate a direct effect of self-disclosure on embodied rapport using Aron's paradigm (H1).

One mechanism through which Aron's paradigm works is through self-expansion, the selfreported broadening of one's self-concept to include another person's beliefs, values, and feelings (Aron, Aron, \& Norman, 2001). If people are indeed psychologically experiencing self-other overlap with another as a result of self-disclosure, then perhaps their behavioral experience is altered as well. That is, if information processing involves one's own motor experience, as suggested by research on embodied cognition, then we speculate that selfdisclosure not only induces "oneness" psychologically but also behaviorally, manifest as synchronized body movements.

In this study, we experimentally induce self-disclosure and use behavioral coding to investigate our second hypothesis that self-disclosure increases behavioral synchrony, which in turn works as a mechanism to facilitate embodied rapport (H2). To our knowledge, this is the first paper to look at behavioral synchrony as it emerges spontaneously in a same-sex stranger-stranger dyadic interaction, with consideration of how it relates to interpersonal connections.

\section{Methods}

\section{Participants}

Ninety-four same-sex participant pairs (41 male: 19 same-race, 22 cross-race; 53 female: 22 same-race, 31 cross-race; $M_{\text {age }}=19.41$ years) were recruited for a study on social coordination across two recruitment waves. Compensation was \$25 in Wave 1 and course credit in Wave 2.

\section{Experimental Procedure}

First, each participant pair was randomly assigned to complete one of two partner interaction tasks, each designed to last for 20 minutes. Pairs randomly assigned to the self-disclosure task completed an abbreviated version of Aron et al.'s (1997) self-disclosure induction paradigm. ${ }^{2}$ Pairs randomly assigned to the control condition read a scientific article 3 together, and took turns indicating and correcting text that required edits to one another. One participant had an "edited" version of the article containing italicizations, strikeouts, and bolded text, and the other participant had an "unedited" version on which to make corrections.

\section{Measures}

Inclusion-of-other in self (IOS; Aron, Aron, \& Smollan, 1992)—Immediately after the partner interaction task, participants each completed questionnaires in private, beginning with a measure of self-other overlap. The IOS is a 7-point single-item scale that visually depicts increasingly overlapping "self" and "partner" circles.

\section{Positive emotions (mDES; Fredrickson, Tugade, Waugh, \& Larkin, 2003)—}

After the IOS scale, participants individually rated 10 different positive emotions categories to indicate how they felt in the interaction (e.g., "love/closeness/trust"; "interested/alert/ curious"; "glad/happy/joyful") using a 9-point scale.

\footnotetext{
${ }^{2}$ The original 45 -minute task consists of self-disclosing items that increase in intensity both within and across three sets of items. We selected from Set I, II, and III, respectively, 4, 4, and 7 items.

${ }^{3}$ Fant, J. B., Kamau, E., \& Preston, C. D. (2005). Chloroplast evidence for the multiple origins of the hybrid potamogeton $\mathrm{x}$ fluitans. Aquatic Botany, 83, 154-160.
} 
Embodied rapport-To assess embodied rapport, after the mDES, participants individually completed Dutton \& Heaphy's (2003) measure of High-quality Connections, comprised of three theoretically-derived subscales $(\alpha=.92)$ : positive regard (e.g., "My partner was friendly and warm toward me"), felt mutuality (e.g., "When I was interacting with my partner, there was a shared flow of thoughts and feelings"), and subjective vitality (e.g., "I felt alive and vital"). Scores were standardized from each subscale and aggregated for each pair.

\section{Behavioral Videocoding Procedure}

Audio and video were simultaneously recorded during the partner interaction task, and a split-screen generator captured one participant on one half of the screen and the other participant in-time on the other half. Two trained coders independently watched the first and last five minutes of all 94 video recordings, scoring both 5-minute segments on the three aspects of behavioral synchrony (Bernieri et al, 1988) using a 7-point semantic differential scale for each aspect: simultaneous movement (nonconcurrent-simultaneous), tempo similarity (dissimilar-similar), and coordination and smoothness (uneasy-smooth). All coding was completed with videos on mute, thereby eliminating influence of audio cues and minimizing demand effects. The coding procedure thus operationalizes behavioral synchrony as a purely physical phenomenon.

For each coder, we first computed two sum scores of the three behavioral synchrony aspects: one for the first five minutes and another for the last five minutes. Because scores for the first and last five minutes were highly correlated $(r=.89$ and .94 for Coders 1 and 2, respectively), we averaged the two sum scores across time points. Inter-rater reliability, calculated by a two-way mixed model (Shrout \& Fleiss, 1979), was high $(\alpha=.87)$.

Accordingly, we averaged the two coders' behavioral synchrony scores to obtain a final behavioral synchrony score for each pair.

\section{Results}

\section{Descriptive Statistics}

Of the 94 pairs considered, 45 were randomly assigned to the self-disclosure task and 49 to the control task. Across both conditions, the mean interaction time was 18.03 minutes $(S D=$ 3.02). See Table 1 for raw means by condition and Table 2 for correlations among pair-level variables.

\section{Manipulation Check}

We fit multilevel models with individuals nested within dyads (Hox, 2002) to assess the impact of the self-disclosure task. Consistent with the self-expansion model (Aron, et al., 2001), results show that self-disclosure condition significantly predicts reported self-other overlap $(b=.62, \mathrm{SE}=.16, p<.01)$. Similarly, consistent with Strong and Aron (2006), results reveal that self-disclosure condition significantly predicts participants' positive emotions $(b=23.64, \mathrm{SE}=2.57, p<.01)$.

\section{A Mediated Model of Self-disclosure and Embodied Rapport}

To test our hypotheses, we conducted bootstrapping analyses following procedures for testing direct and indirect effects (Preacher \& Hayes, 2008). Given past research and results of our manipulation checks, we included feelings of self-other overlap and positive emotions as control variables, each assessed immediately after the interaction task. ${ }^{4}$ Based on a resampling size of 5,000, the bootstrap results indicate that the total direct effect of selfdisclosure condition on embodied rapport $(b=.64, \mathrm{SE}=.32, p<.01)$ decreases to non- 
significance when behavioral synchrony is included as a mediator of the direct effect $(b=$. $19, \mathrm{SE}=.40, p=.63$ ). Even when we partial out the indirect effects of self-other overlap and positive emotions this total indirect effect hypothesized is significant, with a point estimate of .41 and $90 \%$ bootstrap confidence interval that does not include zero $(.02, .38){ }^{5}$ Thus, results are consistent with our hypothesized mediated effect of self-disclosure on embodied rapport via behavioral synchrony, above and beyond subjective feelings of self-other overlap and positive emotions. Beta weights for each pathway of the total indirect effect are shown in Figure $1 .^{6}$ Additional analyses also indicate that the indirect effect does not vary as a function of participant pair gender, racial composition, nor recruitment wave.

\section{Discussion}

This experiment provides the first empirical demonstration of behavioral synchrony's role in fostering embodied rapport. Specifically, stranger-stranger dyads randomly assigned to complete a self-disclosure task, compared to dyads who did not self-disclose, were judged to be moving together more as one orchestrated unit during their social interaction, which in turn predicted higher ratings of positivity, mutuality, and vitality in the interaction.

Noteworthy methods in this study that enrich relationship science include an experimental manipulation of self-disclosure among strangers, and behavioral videocoding of spontaneously emerging behaviors.

Results not only confirm past research on the interpersonal benefits of self-disclosure, but also identify a dynamic and physical component within the process-namely, behavioral synchrony - that works as an independent mechanism to improve social connection quality. As alluded to earlier, our results may be a case of embodied cognition, such that one's behavioral movements are implicated in the affective and psychological experience of a situation. That is, beyond the psychological "we" mindset of intersubjective thoughts and feelings that emerges during interpersonal interactions, there is a coordinated behavioral oneness that also drives how the interaction is experienced. For embodied cognition researchers, behavioral synchrony is a novel starting point to investigate how behaviors of multiple people during a shared experience can shape people's judgments of each other and the situation itself.

\section{Behavioral Synchrony in Various Contexts}

Here, we investigated behavioral synchrony solely under contexts of self-disclosure.

However, in contexts of novel and energizing events with others, such as trying a new sport, taking on a joint business venture, or partaking in a group fitness class (see Aron, Norman, Aron, McKenna, \& Heyman, 2000), relationship quality may also increase to the extent that it also increases behavioral synchrony of the group.

Also, in settings beyond laboratory contexts, understanding behavioral synchrony as a mechanism of interpersonal interactions can be important. The socio-relational development of people with movement disorders (e.g., dyskinesia, Huntington's disease, cerebral palsy),

\footnotetext{
${ }^{4}$ Additional analyses reveal that behavioral synchrony does not mediate the relationship between self-disclosure condition and either self-other overlap or positive emotions, suggesting that each of our control variables is best viewed as a competing process variable that operates during social interaction rather than as an alternative outcome variable. We also position behavioral synchrony as a process variable due to its nonconscious, in-the-moment nature during a conversation. Providing empirical support for this approach, when we entered embodied rapport as a mediator between self-disclosure and behavioral synchrony, less variance is explained $\left(R^{2}=\right.$. $64)$.

${ }^{5}$ Without controlling for self-other overlap and positive emotions, the indirect effect of self-disclosure condition on embodied rapport via behavioral synchrony is also significant (point estimate $=.89 ; 90 \% \mathrm{CI}$ of $.15,1.64$ ).

${ }^{6}$ Standardized beta weights available as supplemental materials. However, caution should be taken in interpreting the standard errors and confidence intervals because although beta weights are standardized, variances of each bootstrap resample are not (Cheung, 2009).
} 
and consequences of technology-mediated communications that reduce the need for face-toface contact, are just two promising research applications.

\section{Closing}

In sum, this study ties together several threads of research to further understand mechanisms for social relationship formation. Although moments of brief social interaction with a stranger may seem irrelevant in the short-term, we suggest that such moments hold potential as "building blocks" for social bonds in the long-term. By unpacking the initial moments of interacting with a stranger, this study stands as a first in identifying behavioral synchrony as a unique ingredient for developing embodied rapport. In moving beyond the individual to consider dyadic interactions holistically, we see that sharing movements is a subtle, yet powerful platform upon which two strangers can create a valued form of "oneness".

\section{Supplementary Material}

Refer to Web version on PubMed Central for supplementary material.

\section{Acknowledgments}

This work was supported by NIMH Grant MH59615.

We thank Sara Algoe, Ben Rosen, Sophie Trawalter, and current PEPLab members for comments on earlier drafts; Michael D. Cohen for data collection and reduction consultation; UNC undergraduates Jana Lembke, Laura Mannie, and Andrew Paschal for videocoding dedication.

\section{References}

Aron, A.; Aron, E.; Norman, CC. Self-expansion model of motivation and cognition in close relationships and beyond. In: Fletcher, GJO.; Clark, M., editors. Blackwell handbook of social psychology: Interpersonal processes. Malden, MA: Blackwell; 2001. p. 478-501.

Aron A, Aron EN, Smollan D. Inclusion of other in the self scale and the structure of interpersonal closeness. Journal of Personality and Social Psychology. 1992; 63:596-612.

Aron A, Melinat E, Aron EN, Vallone RD, Bator RJ. The experimental generation of interpersonal closeness: A procedure and some preliminary findings. Personality and Social Psychology Bulletin. 1997; 23:363.

Aron A, Norman CC, Aron EN, McKenna C, Heyman RE. Couples's shared participation in novel and arousing activities and experienced relationship quality. Journal of Personality and Social Psychology. 2000; 78:273-284. [PubMed: 10707334]

Bernieri FJ, Reznick JS, Rosenthal R. Synchrony, pseudosynchrony, and dissynchrony: Measuring the entrainment process in mother-infant interactions. Journal of Personality and Social Psychology. 1988; 54:243-253.

Bernieri FJ. Coordinated movement and rapport in teacher-student interactions. Journal of Nonverbal Behavior. 1988; 12:120-138.

Brown SL, Fredrickson BL, Wirth MM, Poulin MJ, Meier EA, Heaphy ED, et al. Social closeness increases salivary progesterone in humans. Hormones and Behavior. 2009; 56:108-111. [PubMed: 19362559]

Cohen EEA, Ejsmond-Frey R, Knight N, Dunbar RIM. Rowers' high: Behavioural synchrony is correlated with elevated pain thresholds. Biology Letters. 2010; 6:106-108. [PubMed: 19755532]

Collins NL, Miller LC. Self-disclosure and liking: A meta-analytic review. Psychological Bulletin. 1994; 116:457-457. [PubMed: 7809308]

Condon WS, Sander LW. Neonate movement is synchronized with adult speech: Interactional participation and language acquisition. Science. 1974; 183:99. [PubMed: 4808791] 
Dutton, JE.; Heaphy, ED. The power of high-quality connections. In: Cameron, KS.; Dutton, JE.; Quinn, RE., editors. Positive organizational scholarship: Foundations of a new discipline. San Francisco, CA: Berrett-Koehler; 2003. p. 263-278.

Feldman R. Parent-infant synchrony: Biological foundations and developmental outcomes. Current Directions in Psychological Science. 2007; 16:340-345.

Fredrickson BL, Tugade MM, Waugh CE, Larkin GR. What good are positive emotions in crisis? A prospective study of resilience and emotions following the terrorist attacks on the United States on September $11^{\text {th }}, 2001$. Journal of Personality and Social Psychology. 2003; 84:365-376. [PubMed: 12585810]

Hove MJ, Risen JL. It's all in the timing: interpersonal synchrony increases affiliation. Social Cognition. 2009; 27:949-960.

Hox, JJ. Multilevel analysis: Techniques and applications. Mahwah, NJ: Lawrence Erlbaum Associates; 2002.

Jones SM, Wirtz JG. "Sad monkey see, monkey do": Nonverbal matching in emotional support encounters. Communication Studies. 2007; 58:71-86.

Jourard SM. Self-disclosure and other-cathexis. Journal of Abnormal and Social Psychology. 1959; 59:428-431. [PubMed: 14407982]

Kashdan TB, Roberts JE. Social anxiety's impact on affect, curiosity, and social self-efficacy during a high self-focus social threat situation. Cognitive Therapy and Research. 2004; 28:119-141.

Kimura M, Daibo I. Interactional synchrony in conversations about emotional episodes: A measurement by "the between-participants pseudosynchrony experimental paradigm". Journal of Nonverbal Behavior. 2006; 30:115-126.

McNeill, WH. Keeping together in time: Dance and drill in human history. Cambridge: Harvard University Press; 1995.

Niedenthal P. Embodying emotion. Science. 2007; 316:1002-1005. [PubMed: 17510358]

Preacher KJ, Hayes AF. Asymptotic and resampling strategies for assessing and comparing indirect effects in multiple mediator models. Behavior Research Methods. 2008; 40:879. [PubMed: 18697684]

Shrout PE, Fleiss JL. Intraclass correlations: uses in assessing rater reliability. Psychological Bulletin. 1979; 86:420-428. [PubMed: 18839484]

Strong, G.; Aron, A. The effect of shared participation in novel and challenging activities on experienced relationship quality: Is it mediated by high positive affect?. In: Vohs, KD.; Finkel, EJ., editors. Self and relationships: Connecting intrapersonal and interpersonal processes. New York, NY: Guilford; 2006. p. 342-359.

Snyder, CR.; Lopez, SJ. Handbook of positive psychology. New York, NY: Oxford University Press; 2002.

Tickle-Degnen L, Rosenthal R. The nature of rapport and its nonverbal correlates. Psychological Inquiry. 1990; 1:285-293.

Valdesolo P, DeSteno D. Synchrony and the social tuning of compassion. Emotion. 2011; 11:262-266. [PubMed: 21500895]

Weinberg MK, Tronick EZ. Infant affective reactions to the resumption of maternal interaction after the still-face. Child Development. 1996; 67:905-914. [PubMed: 8706534]

Wiltermuth SS, Heath C. Synchrony and cooperation. Psychological Science. 2009; 20:1. [PubMed: 19152536] 


\section{Research Highlights}

- Synchrony is observed as a behavioral process variable in relationship formation.

- Dyads of strangers in a manipulated context of self-disclosure:

- spontaneously display more synchrony and report more embodied rapport.

- Synchrony mediates the direct effect of self-disclosure on embodied rapport.

- Results control for indirect effects of self-other overlap and positive affect. 


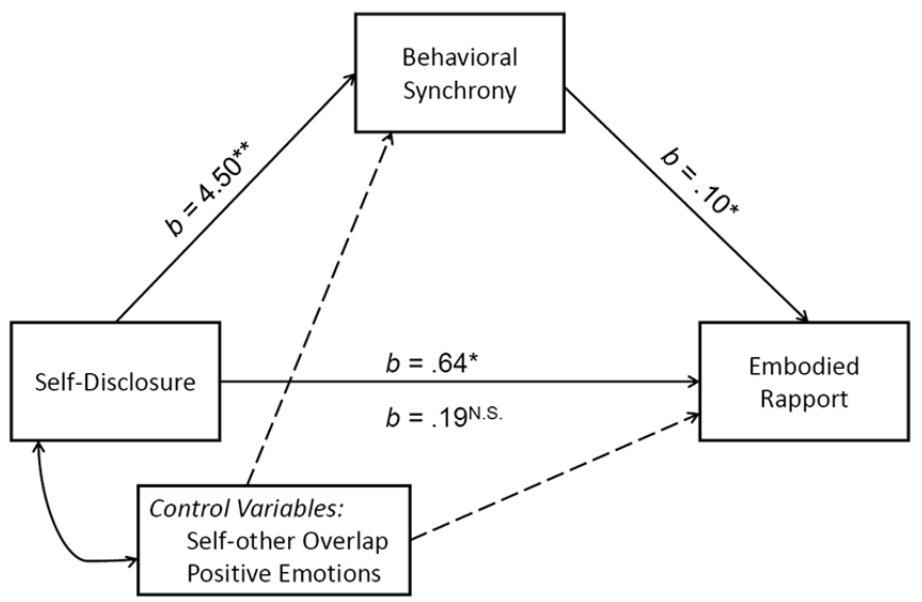

Figure 1.

Mediated model for self-disclosure, behavioral synchrony, and embodied rapport. Statistics for direct effect are above path, and total indirect effect below path. Results from bootstrap analyses (resample size $=5,000$ ) signify significant mediation, controlling for self-other overlap and positive emotions. ${ }^{*} p<.01 .{ }^{*} p<.05$. Reported tests are one-tailed. $\mathrm{R}^{2}=.78$. 
Table 1

Descriptive Statistics of Individual- and Pair-Level Variables across Conditions

\begin{tabular}{lccccc}
\hline & \multicolumn{4}{c}{ Control Task } & \multicolumn{3}{c}{ Self-Disclosure Task } \\
\cline { 2 - 6 } & $\mathbf{n}$ & $\boldsymbol{M}$ & $\mathbf{n}$ & $\boldsymbol{M}$ & $\boldsymbol{t}$ \\
\hline $\begin{array}{l}\text { Individual-level Variables } \\
\text { Self-other Overlap }{ }^{\dagger * *}\end{array}$ & 101 & $2.64(.97)$ & 85 & $3.26(1.03)$ & - \\
Positive Emotions ${ }^{\dagger * *}$ & 102 & $38.93(15.20)$ & 85 & $62.59(18.18)$ & - \\
\hline $\begin{array}{l}\text { Dyad-level Variables } \\
\text { Observed Behavioral Synchrony }\end{array}$ & 49 & $8.39(1.38)$ & 45 & $13.43(2.53)$ & $12.09^{* *}$ \\
$\quad$ Embodied Rapport & 49 & $-1.45(1.77)$ & 45 & $1.60(1.40)$ & $9.19^{* *}$ \\
\hline
\end{tabular}

Note. Standard deviations presented in parentheses. Missing individual-level data used for manipulation checks do not influence main analysis.

${ }^{\dagger}$ significantly predicted by experimental condition using multilevel modeling.

$* *$

$p<.01$. Reported tests are one-tailed. 
Table 2

Correlations among Pair-Level Study Variables

\begin{tabular}{lcccc}
\hline Variable & $\mathbf{1 .}$ & $\mathbf{2 .}$ & $\mathbf{3 .}$ & $\mathbf{4 .}$ \\
\hline 1. Self-other Overlap & - & & & \\
2. Positive Emotions & $.57^{* *}$ & - & & \\
3. Observed Behavioral Synchrony & $.39^{* *}$ & $.62^{* *}$ & - & \\
4. Embodied Rapport & $.51^{* *}$ & $.87^{* *}$ & $.64^{* *}$ & - \\
\hline Note. & & & & \\
$\begin{array}{l}* * \\
p<.01 . \mathrm{n}=94 .\end{array}$ & & & &
\end{tabular}

